\title{
Psychotherapy mediated by remote communication technologies: a meta-analytic review
}

\author{
Penny E Bee*1, Peter Bower ${ }^{2}$, Karina Lovell1', Simon Gilbody ${ }^{3}$, \\ David Richards ${ }^{3}$, Linda Gask² and Pamela Roach ${ }^{1}$
}

\begin{abstract}
Address: ${ }^{1}$ School of Nursing, Midwifery and Social Work, University of Manchester, Oxford Road, Manchester, M13 9PL, UK, ${ }^{2}$ National Primary Care Research And Development Centre, University of Manchester, Oxford Road, Manchester, M13 9PL, UK and ${ }^{3}$ Department of Health Sciences, University of York, University Road, Heslington, York, YO10 5DD, UK

Email: Penny E Bee* - Penny.Bee@manchester.ac.uk; Peter Bower - Pete.Bower@manchester.ac.uk; Karina Lovell - Karina.Lovell@manchester.ac.uk; Simon Gilbody - sg519@york.ac.uk; David Richards - dr17@york.ac.uk; Linda Gask - Linda.Gask@manchester.ac.uk; Pamela Roach - Pamela.Roach@manchester.ac.uk

* Corresponding author
\end{abstract}

Published: 22 July 2008

BMC Psychiatry 2008, 8:60 doi:10.1/86/147|-244X-8-60
Received: 28 January 2008

Accepted: 22 July 2008

This article is available from: http://www.biomedcentral.com/I47I-244X/8/60

(c) 2008 Bee et al; licensee BioMed Central Ltd.

This is an Open Access article distributed under the terms of the Creative Commons Attribution License (http://creativecommons.org/licenses/by/2.0), which permits unrestricted use, distribution, and reproduction in any medium, provided the original work is properly cited.

\begin{abstract}
Background: Access to psychotherapy is limited by psychopathology (e.g. agoraphobia), physical disability, occupational or social constraints and/or residency in under-served areas. For these populations, interventions delivered via remote communication technologies (e.g. telephone, internet) may be more appropriate. However, there are concerns that such delivery may influence the therapeutic relationship and thus reduce therapy effectiveness. This review aimed to determine the clinical effectiveness of remotely communicated, therapist-delivered psychotherapy.
\end{abstract}

Methods: Systematic review (including electronic database searching and correspondence with authors) of randomised trials of individual remote psychotherapy. Electronic databases searched included MEDLINE (1966-2006), Psyclnfo (1967-2006), EMBASE (1980-2006) and CINAHL databases (1982-2006). The Cochrane Central Register of Controlled Trials (CENTRAL) and the Cochrane Collaboration Depression, Anxiety and Neurosis Controlled Trials Register (CCDANCTR). All searches were conducted to include studies with a publication date to July 2006.

Results: Thirteen studies were identified, ten assessing psychotherapy by telephone, two by internet and one by videoconference. Pooled effect sizes for remote therapy versus control conditions were 0.44 for depression $(95 \% \mathrm{Cl} 0.29$ to $0.59,7$ comparisons, $\mathrm{n}=726)$ and 1.15 for anxiety-related disorders $(95 \% \mathrm{Cl} 0.8 \mathrm{I}$ to I.49, 3 comparisons, $\mathrm{n}=168)$. There were few comparisons of remote versus face-to-face psychotherapy.

Conclusion: Remote therapy has the potential to overcome some of the barriers to conventional psychological therapy services. Telephone-based interventions are a particularly popular research focus and as a means of therapeutic communication may confer specific advantages in terms of their widespread availability and ease of operation. However, the available evidence is limited in quantity and quality. More rigorous trials are required to confirm these preliminary estimates of effectiveness. Future research priorities should include overcoming the methodological shortcomings of published work by conducting large-scale trials that incorporate both clinical outcome and more process-orientated measures. 


\section{Background}

Psychological disorders account for over 15\% of the total burden of disease within established economies, a significant proportion of which manifests in depressive and anxiety-related disorders [1]. For these disorders, effective treatment options often include non-pharmacological as well as pharmacological interventions. Consensus guidelines recommend the use of cognitive-behavioral and interpersonal therapies for major depression [2-4] and cognitive behavioral therapy for panic disorder [5]. Research has also highlighted the potential value of these approaches in the treatment of dysthymia, phobia and generalized anxiety disorder [6].

Despite evidence of its efficacy however, most adults diagnosed with depressive or anxiety-related disorders do not receive psychotherapeutic care [6,7]. Insufficient numbers of mental health professionals impede access to effective interventions, with people living in remote or underserved areas often having to travel long distances to obtain face-to-face services [8]. Other recognized barriers include time and economic constraints, caring responsibilities, psychological or physical impairment and concerns regarding the potential stigma of attending outpatient appointments [9-11].

Remote communication technologies such as the telephone, internet or videophone have the potential to mitigate many of these inequalities $[12,13]$. The telephone in particular is a widely available telecommunication technology [14] that is being used increasingly as a mechanism for support and treatment delivery $[15,16]$. The establishment of 1-900 counseling services alone illustrates a long but ongoing commitment to its use within psychotherapy service provision. More recently, the emergence of computer-aided technology alongside growth in the popularity of the Internet have increased multifold the opportunities for real time, long-distance consultations [17].

Conventional wisdom still insists however, that for most purposes, psychological therapies should be delivered face-to-face. The central premise to this argument is that the effectiveness of such interventions depends upon on the development of a high quality therapeutic alliance between therapist and client [18]. Within the context of this relationship, visual as well as auditory information is reflected in behaviours such as eye contact, physical expression, posture and voice [19]. The use of the telephone or internet will invariably eliminate many of these cues whilst the use of teleconferencing will limit physical presence and touch. Whether or not an effective alliance can be delivered in the absence of interpersonal contact thus remains a topic of some debate [20-22].
Evidence to support the efficacy of non face-to-face psychotherapy service models is accumulating. Recent studies of collaborative care provision, in which telephone support is often provided as an adjunct to other interventions, have documented positive outcomes [23-27]. Reviews and meta-analyses of computer-delivered CBT packages $[28,29]$ and self-help treatments [30-33] also provide promising results. These interventions however are often considered as stand-alone technologies requiring little or no contact between therapist and client. One recent metaanalysis of internet-based cognitive behavioral therapy for depression and anxiety [34] has suggested that the efficacy of these psychological interventions may ultimately be higher where therapist support is available.

The ability to deliver more intensive psychological therapy via remote communication media has the potential to confer multiple benefits for patients by combining real time, scheduled contact with increased accessibility. As yet however, the efficacy of delivering psychotherapy via such means has received comparatively little attention [19]. This article reports the findings of a systematic review conducted to determine the clinical effectiveness of remote psychotherapy.

\section{Methods \\ Design}

This research was conducted using systematic review techniques [35] and meta-analysis to assess the clinical effectiveness of psychotherapy delivered via remote communication.

\section{Inclusion criteria \\ Study design}

Studies eligible for inclusion were published randomised controlled trials (RCTs). Due to a lack of financial resources for translation facilities, the review was restricted to English language publications. There were no specific methodological quality criteria for inclusion in the review. Instead data were extracted from all studies on key methodological issues (see 'Methods of the review' below).

\section{Interventions}

Interventions eligible for inclusion included any treatment incorporating a psychological intervention mediated by remote communication. Psychological interventions were defined as treatments with an explicit psychological orientation [36]. 'Mediated by remote communication' was defined as a treatment where all or the majority (i.e. with the exception of an initial or final contact meeting) of the psychological intervention was delivered by a therapist to a patient on a scheduled and repeated one-to-one basis through an appropriate technology (rather than face-to-face). Group and marital ther- 
apies or any other intervention involving interaction between a therapist and more than one client simultaneously were excluded from the review. Trials examining emergency crisis interventions (e.g. telephone helplines) or the efficacy of medication management programs in the absence of psychotherapy were excluded. Minimal interventions in which the majority of therapy was delivered i) outside of client-therapist interaction time (e.g. self-help therapy) or ii) via technology with little or no therapist contact (e.g. computerized CBT) were also excluded. Studies measuring changes in mental health symptoms as a by-product of counseling for physical illness were excluded.

\section{Populations}

Populations eligible for inclusion included any group seeking treatment for a mood disorder or functional (nonorganic) mental health problem recognized by ICD-10 [37] or DSM criteria [38]. Studies involving therapy for substance misuse or addictions were excluded. Trials examining the efficacy of remote therapy for healthy populations at risk of mental health difficulties were also excluded.

\section{Search strategy}

Electronic literature searches were performed using the Ovid electronic database on the MEDLINE (1966-2006), PsycInfo (1967-2006), EMBASE (1980-2006) and CINAHL databases (1982-2006). The Cochrane Central Register of Controlled Trials (CENTRAL) and the Cochrane Collaboration Depression, Anxiety and Neurosis Controlled Trials Register (CCDAN-CTR) were also searched. All searches were conducted to July 2006. Subject headings were used to identify all papers indexed as containing material relevant to mental/psychiatric health, psychological therapies/interventions and communication technologies. These headings were augmented by text words that included the full and abbreviated names of specific mental disorders, types of psychological therapy and modes of communication. Full details of the search terms used are appended.

Authors of published and ongoing studies were contacted for further studies and information on the progress of ongoing work: $50 \%$ replied with information. Reference lists from identified papers and from reviews in the area were searched by hand.

\section{Methods of the review}

Eligibility judgements and data extraction were done independently by two reviewers. No formal measure of the reliability of data extraction was calculated but disagreements were resolved by discussion with other members of the project team.
A standardised data extraction recorded information on study context, population, interventions, outcomes and methodological quality. Methodological quality was assessed using standard criteria originally developed by the Cochrane Collaboration for Depression, Anxiety and Neurosis (CCDAN). The CCDAN quality rating scale [39] scores each study according to 23 elements of design and conduct including randomisation methods, sample size, follow-up period, power and appropriateness of analysis. Each criterion is scored from 0-2, giving a maximum score of 46 .

The CCDAN criteria, like other validated scales of RCT quality [40], include a score pertaining to the adequacy of blinding procedures. Since it is not feasible to blind patients to an active intervention, this criterion was not relevant to the present review. A second criterion pertaining to the recording of pharmacological side-effects was similarly omitted from the review. Thus overall quality scores for the CCDAN criteria were in this study only able to reach a maximum of 42 .

\section{Methods of analysis}

All analyses were conducted using Comprehensive Meta Analysis [41]. Continuous measures were translated into a standardised effect size, Cohen's $d$ [42]. Dichotomous variables were translated into standardised effect sizes by computing the log odds ratio, and then conversion to $d$ using procedures in the meta analytic package. Initial meta-analyses used a fixed effect model [43] to provide an overall pooled measure of effect. Between-study heterogeneity was assessed using the $\mathrm{I}^{2}$ statistic [44], which describes the percentage of total variation across studies that is due to heterogeneity rather than chance. The $\mathrm{I}^{2}$ statistic has several advantages over other measures of heterogeneity, including greater statistical power to detect clinical heterogeneity when fewer studies are available. As a guide, $\mathrm{I}^{2}$ values of $25 \%$ may be considered 'low', 50\% 'moderate' and 75\%, 'high'. Where heterogeneity was 'high' (i.e. $\mathrm{I}^{2}$ values of $75 \%$ or above), a random effects model was employed.

For the purposes of analysis, three main comparison groups were created: i) remote communication therapy versus a control group (e.g. usual care, waiting list); ii) remote communication therapy versus conventional faceto-face therapy, and iii) different types of remotely communicated therapy. Where studies compared two different types of remote therapy with a control, both comparisons were entered into the meta-analysis separately, but control group sample sizes were halved to ensure that they were not double counted [45].

Studies examining depression underwent a separate analysis to those focussing on anxiety or anxiety-related disor- 
ders (e.g. anxiety, agoraphobia with panic, OCD). Studies assessed outcome over a range of time points. For the purposes of analysis, outcome assessments were categorised into two time periods, representing outcomes in the short (0-6 months), and longer term (7 months and over). Where studies reported assessments at multiple time points, data with the longest duration were used. Effect sizes were only calculated for the primary outcome measure (where specified), or the measure deemed most relevant to the mental health disorder under study (judged by $\mathrm{KL}$ and LG). When studies reported more than one relevant measure (e.g. BDI and HRSD), these were combined by taking an arithmetic mean [46].

\section{Results}

Thirteen studies were included in the review. Of 40 studies identified but subsequently excluded, the most common reasons for rejection were $\mathrm{i}$ ) a lack of a mental health primary outcome measure $(n=11)$; ii) the use of a minimal intervention (e.g. guided self help) or an intervention with no clear psychological orientation $(\mathrm{n}=10)$; iii) the use of a technology-based therapy requiring no direct therapist involvement $(n=18)$ or iv) group therapy $(n=$ 1 ). One further study was identified as ongoing and this may be of relevance for later versions of the review. Summaries of the included studies are given in table 1, with corresponding methodological details in table 2. A list of excluded studies is available from the authors.

\section{Scope of the included studies}

Nine studies reported 11 comparisons of technologymediated therapy versus a control, three studies focussing on anxiety-related disorders [47-49] and six on depression [50-55]. Four of these studies used usual care as the comparative arm and four a waiting list or no treatment control. The remaining study did not provide a clear description of its control group. Two studies reported comparisons of technology-mediated therapy with conventional face-to-face interventions, one in an anxietyrelated disorders (OCD) [56] and one in depression [57]. Three studies reported comparisons between different types of therapy mediated by the same technology, one in anxiety [58] and three in depression $[54,55,59]$.

Ten studies assessed the efficacy of psychological therapy mediated by telephone, two by internet and one by videoconference (Table 1). Participants were recruited via primary care screening or GP referral (five studies), secondary care outpatient screening (one study), by public advertisement (two studies), through case registers of Multiple Sclerosis patients (two studies), via longitudinal research (one study) and by access to a pool of psychology students (one study). One study focusing on depressed children did not provide a clear description of its recruitment context. Patients included those with PTSD, OCD, agoraphobia with panic disorder and depression (Table 2 ). The number of participants randomized within each trial ranged from 23 to 600 (mean 121), with losses at follow-up of between $9 \%$ and $73 \%$. Length of follow-up ranged from 6 weeks to 1 year. No data on costs were reported in any of the studies.

\section{Quality of the included studies}

All studies were RCTs, although in two studies methods of allocation were only quasi-randomised with the possibility of bias [50,58]. Only two out the 13 studies reported details of allocation concealment $[55,56]$. Less than half of the included studies $(\mathrm{n}=5)$ defined a main outcome $a$ priori $[50,51,54-56]$ and only five reported conducting a power analysis [49,54-57]. Although all studies conducted largely or wholly appropriate analyses of their data this failed to include an intention to treat analysis in eight instances [47-51,54,57,58]. Seven studies controlled for between-group differences at baseline $[47,48,51,53,55,56,59]$ and all but two studies presented sufficient data for re-analysis $[50,57]$. Only two studies involved more than 100 participants per trial arm $[51,55]$, the vast majority $(n=9)$ recruiting less than 50 .

The mean methodological quality score for the included studies was $23(\mathrm{SD}=7.33)$. The highest scores were 38 [55], 36 [56] and 31 [59].

\section{Quantitative results}

Pooled effect sizes for each of the main comparison groups are presented in table 3 . In both meta analyses using multiple studies, the $\mathrm{I}^{2}$ statistic was zero (indicating no heterogeneity beyond that expected by chance) and thus fixed effect models were used.

For depressive disorders, the pooled effect size for remotely delivered therapy compared to control conditions (e.g. waiting list or usual care) was 0.44 (95\% CI 0.29 to 0.59 ), demonstrating a statistically significant 'medium' effect [42] (figure 1).

For anxiety-related disorders, remotely delivered therapy demonstrated a 'large' effect compared to control conditions (figure 2, 3 comparisons, $\mathrm{n}=168$ ). The pooled effect size was 1.15 ( $95 \%$ CI 0.81 to 1.49 ) (figure 2).

Comparisons between technology-mediated and face-toface interventions and comparisons between different forms of technology-mediated therapy were limited by the small number of studies reporting relevant data (table $3)$.

\section{Discussion}

The aim of this review was todetermine the clinical effectiven ess of psychotherapy delivered by remote communi- 
Table I: Interventions in the review

\begin{tabular}{|c|c|c|c|}
\hline Study & Target population & Study groups & Description of intervention in each group \\
\hline \multirow[t]{3}{*}{ Hunkeler (2000) } & Depressed primary care patients & $\begin{array}{l}\text { Usual care plus telephone } \\
\text { support \& peer care }\end{array}$ & $\begin{array}{l}\text { 'Good care' incorporating regular GP visits, } \\
\text { continued antidepressant prescribing and any other } \\
\text { referral thought usual by GP. Augmented by } \\
\text { telephone-delivered medication adherence support, } \\
\text { side-effect discussions and behavioural activation } \\
\text { plans (mean of } 10.1 \times 5.6 \text { min sessions over } 16 \mathrm{wks}) \\
\text { plus one or more telephone or face-to-face }(6 / 62 \\
\text { participants) peer support contacts. }\end{array}$ \\
\hline & & $\begin{array}{l}\text { Usual care plus telephone } \\
\text { support }\end{array}$ & As above, minus peer support \\
\hline & & Usual care & As above minus telephone \& peer support. \\
\hline
\end{tabular}

\begin{tabular}{lll}
\hline Lange (200I) $\quad \begin{array}{l}\text { Psychology students with trauma } \\
\text { experience }\end{array}$ & $\begin{array}{l}\text { Internet-mediated writing } \\
\text { therapy }\end{array}$
\end{tabular}

30 web-pages of psychoeducation followed by $10 \times$ experience therapy 45-min writing sessions over 5 wks (2/wk), therapist feedback (approx 450 words) provided on 7 occasions across 3 treatment phases (selfconfrontation, cognitive re-appraisal, sharing \& farewell ritual).

Waiting list $\quad 30$ web pages of psychoeducation only

\begin{tabular}{|c|c|c|c|}
\hline \multirow[t]{2}{*}{ Lange (2003) } & $\begin{array}{l}\text { Individuals with mild-relatively } \\
\text { severe trauma symptoms }\end{array}$ & $\begin{array}{l}\text { Internet-mediated writing } \\
\text { therapy }\end{array}$ & $\begin{array}{l}30 \text { web-pages of psychoeducation followed by } 10 \times \\
45 \text {-min writing sessions over } 5 \text { wks }(2 / \text { wk), therapist } \\
\text { feedback (approx } 450 \text { words) provided on } 7 \\
\text { occasions across } 3 \text { treatment phases (self- } \\
\text { confrontation, cognitive re-appraisal, sharing \& } \\
\text { farewell ritual). }\end{array}$ \\
\hline & & Delayed treatment & $\begin{array}{l}\text { As above, but only received once the intervention } \\
\text { group had completed treatment. }\end{array}$ \\
\hline
\end{tabular}

\begin{tabular}{ll}
\hline Lovell (2006) & Secondary care outpatients with \\
OCD & Face-to-face CBT
\end{tabular}

$10 \times 1-h r$ sessions using exposure \& response prevention. Sessions incorporated the establishment of fear hierarchies, use of family co-therapist, weekly exposure targets (to be practised between sessions for at least I-hr/dy), homework reviews and collaborative problem solving.

Telephone CBT 8 weekly telephone calls of up to 30 -mins in length with treatment content identical to above. Homework sheets posted to participants. Initial Ihr face-to-face session covering the same material as the face-to-face arm plus I $\times$ I-hr final session face-to-face

\begin{tabular}{ll}
\hline Lynch (1997) Primary care patients with minor $\quad$ Telephone counselling \\
depression
\end{tabular}

$6 \times 20$-min sessions based on problem-solving for depression; homework comprising of 5 steps of treatment including a demonstration of the connection between depressed mood and problems, expressing problems in a form that facilitates solutions, evaluating and modifying these solutions.

Comparison group No further details provided

\begin{tabular}{|c|c|c|c|}
\hline \multirow[t]{3}{*}{ Lynch (2004) } & $\begin{array}{l}\text { Primary care patients with minor } \\
\text { depression }\end{array}$ & Telephone problem solving & $\begin{array}{l}\text { Nezu's problem solving therapy adapted for } \\
\text { telephone use and administered over a } 6 \text {-wk period }\end{array}$ \\
\hline & & Telephone stress management & $\begin{array}{l}\text { Treatment designed to serve as an attention control } \\
\text { with topics including the identification of sources of } \\
\text { stress, the importance of diet \& exercise, ways of } \\
\text { coping with stress }\end{array}$ \\
\hline & & Usual care & $\begin{array}{l}\text { Usual treatment deemed appropriate by primary } \\
\text { care physician. }\end{array}$ \\
\hline
\end{tabular}


Table I: Interventions in the review (Continued)

\begin{tabular}{|c|c|c|c|}
\hline \multirow[t]{2}{*}{ McName e (1989) } & \multirow[t]{2}{*}{$\begin{array}{l}\text { Housebound agoraphobics with } \\
\text { panic disorder }\end{array}$} & Telephone self exposure & $\begin{array}{l}\text { Exposure goals set via } 10 \times 12 \text {-min telephone } \\
\text { contacts with therapists. Subjects posted a self-help } \\
\text { manual that encouraged use of coping strategies and } \\
\text { family co-therapists. }\end{array}$ \\
\hline & & Telephone relaxation therapy & $\begin{array}{l}\text { Subjects posted standard taped instructions of } \\
\text { Jacobsen's relaxation and instructed to listen for at } \\
\text { least I-hr/dy. Therapy augmented by } 10 \times 12 \text {-min } \\
\text { telephone consultations. }\end{array}$ \\
\hline \multirow[t]{2}{*}{ Miller (2002) } & $\begin{array}{l}\text { Women with history of recurrent/ } \\
\text { chronic depression }\end{array}$ & $\begin{array}{l}\text { Telephone interpersonal } \\
\text { psychotherapy (IPT-T) }\end{array}$ & I2 $\times$ I-hr scheduled weekly sessions. \\
\hline & & Usual care & No treatment beyond usual care \\
\hline \multirow[t]{2}{*}{ Mohr (2000) } & Depressed MS patients & Telephone CBT & $\begin{array}{l}8 \times 50 \text {-min sessions plus a workbook with } \\
\text { assignments. Treatment delivered alongside access } \\
\text { to usual care. }\end{array}$ \\
\hline & & Usual care & $\begin{array}{l}\text { Any treatment given in the course of usual clinician } \\
\text { care. }\end{array}$ \\
\hline \multirow[t]{2}{*}{ Mohr (2005) } & $\begin{array}{l}\text { Depressed primary care patients } \\
\text { with MS }\end{array}$ & Telephone CBT (T-CBT) & Weekly 50 -min sessions completed over 16 wks. \\
\hline & & $\begin{array}{l}\text { Telephone supportive emotion } \\
\text { focussed therapy (T-SEFT) }\end{array}$ & Weekly 50 -min sessions completed over 16 wks \\
\hline \multirow[t]{2}{*}{ Nelson (2003) } & Depressed children aged 8-14 yrs & Videoconferenc e CBT & 8 sessions $(I \times 90$-min plus $7 \times 60-\mathrm{min})$ \\
\hline & & Face-to-face CBT & 8 sessions $(1 \times 90-\mathrm{min}$ plus $7 \times 60-\mathrm{min})$ \\
\hline \multirow[t]{3}{*}{ Simon (2004) } & Depressed primary care patients & Telephone psychotherapy & $\begin{array}{l}8 \times 30-40 \text { min CBT plus I mail contact and } 3 \times 10- \\
15 \text { min telephone sessions focussed on medication } \\
\text { management, caseload tracking and structured } \\
\text { assessment. }\end{array}$ \\
\hline & & Telephone care management & $\begin{array}{l}\text { As above minus telephone CBT. Patients given CBT } \\
\text { self-management booklet but no further support } \\
\text { provided. }\end{array}$ \\
\hline & & Usual care & No further details given \\
\hline \multirow[t]{2}{*}{ Swinson (1995) } & $\begin{array}{l}\text { Rural primary care patients suffering } \\
\text { from panic disorder with } \\
\text { agoraphobia }\end{array}$ & Telephone behaviour therapy & $\begin{array}{l}\text { Mailed psychometric package and educational } \\
\text { workbook serving as an introduction to behavior } \\
\text { therapy concepts (e.g. hierarchy construction, } \\
\text { exposure exercises, record keeping); } 8 \times 1 \text {-hr } \\
\text { scheduled therapy sessions completed over approx. } \\
10 \text { wks. Therapy included exposure principles \& } \\
\text { exercises, long term goals, hierarchy construction, } \\
\text { coping strategies, diary keeping, homework planning } \\
\text { \& reviewing. }\end{array}$ \\
\hline & & Waiting list & $\begin{array}{l}\text { Initial psychometric package followed } 10 \text { wks later } \\
\text { by an additional psychometric package and a } \\
\text { workbook serving as an introduction to behavior } \\
\text { therapy concepts (e.g. hierarchy construction, } \\
\text { exposure exercises, record keeping). }\end{array}$ \\
\hline
\end{tabular}

cation technologies. Compared to control conditions, technology-mediated therapy demonstrated a 'large' effect for anxiety-related disorders and a 'medium' effect for depression. The majority of included studies suffered at least some methodological limitations and few comparisons of remote versus face-to-face psychotherapy were found.

Overall, the effect size calculations would suggest that remotely delivered psychotherapies do have the potential to be clinically effective, although the magnitude of this effect varies and may be more evident for anxiety than for depression. The comparison of technology-mediated psychotherapy against control conditions for depression produced a pooled effect size of 0.44 in favor of therapy. This finding remains in accordance with an effect size of 0.42 reported by an earlier meta-analysis [60] comparing conventional face-to-face therapy with no treatment controls in depression. A second more recent meta-analysis of faceto-face psychotherapy for depression reports standardized mean differences of 0.63 up to 3 months and 0.56 at $6-9$ 
Table 2: Characteristics of the included studies

\begin{tabular}{|c|c|c|c|c|c|c|c|c|}
\hline Study & Country & $\begin{array}{l}\text { Target } \\
\text { population }\end{array}$ & Recruitment & $\begin{array}{l}\text { Sample } \\
\text { size }\end{array}$ & Outcomes & Follow-up & $\begin{array}{l}\text { Follow-up } \\
\text { rate }\end{array}$ & $\begin{array}{l}\text { CCDAN } \\
\text { score }\end{array}$ \\
\hline $\begin{array}{l}\text { Hunkele } r \\
(2000)\end{array}$ & US & $\begin{array}{l}\text { Depressed } \\
\text { primary care } \\
\text { patients }\end{array}$ & GP referral & 302 & $\begin{array}{l}\text { HAMD, BDI, } \\
\text { SF-I } 2\end{array}$ & $\begin{array}{l}\text { Baseline, } 6 \mathrm{w}, 6 \\
\mathrm{~m}\end{array}$ & $\begin{array}{l}90 \% \text { at } 6 w, \\
85 \% \text { at } 6 \mathrm{~m}\end{array}$ & 25 \\
\hline Lange (200I) & Netherlands & $\begin{array}{l}\text { Psychology } \\
\text { students with } \\
\text { trauma experience }\end{array}$ & $\begin{array}{l}\text { From student } \\
\text { pool in return for } \\
\text { course credits }\end{array}$ & 30 & $\begin{array}{l}\text { IES, SCL-90, } \\
\text { POMS }\end{array}$ & $\begin{array}{l}\text { Baseline, } 5 \mathrm{w}, \mathrm{II} \\
\mathrm{w}\end{array}$ & $\begin{array}{l}83 \% \text { at } 5 w \\
27 \% \text { at } 11 \mathrm{w}\end{array}$ & 18 \\
\hline Lange (2003) & Netherlands & $\begin{array}{l}\text { Individuals with } \\
\text { mild-relatively } \\
\text { severe trauma } \\
\text { symptoms }\end{array}$ & Website contact & 184 & IES, SCL-90 & $\begin{array}{l}\text { Baseline, } 5 \text { w, II } \\
\text { w }\end{array}$ & $\begin{array}{l}79 \% \text { at } 5 w, \\
31 \% \text { at } I I w\end{array}$ & 21 \\
\hline Lovell (2006) & UK & $\begin{array}{l}\text { Secondary care } \\
\text { outpatients with } \\
\text { OCD }\end{array}$ & Screening clinics & 72 & YBOCs, BDI & $\begin{array}{l}\text { Pre-baseline, } \\
\text { baseline, I m, } 3 \\
\text { m, } 6 \mathrm{~m}\end{array}$ & $90 \%$ at $6 \mathrm{~m}$ & 36 \\
\hline Lynch (1997) & US & $\begin{array}{l}\text { Primary care } \\
\text { patients with } \\
\text { minor depression }\end{array}$ & Screening & 29 & $\begin{array}{l}\text { BDI, HAMD, } \\
\text { DHP, PSI }\end{array}$ & Baseline, $7 \mathrm{w}$ & $55 \%$ at $7 w$ & 20 \\
\hline Lynch (2004) & US & $\begin{array}{l}\text { Primary care } \\
\text { patients with } \\
\text { minor depression }\end{array}$ & Screening & 54 & $\begin{array}{l}\text { BDI, HAMD, } \\
\text { DHP }\end{array}$ & Baseline, $6 \mathrm{w}$ & $57 \%$ at $6 w$ & 17 \\
\hline $\begin{array}{l}\text { McNam ee } \\
(1989)\end{array}$ & UK & $\begin{array}{l}\text { Housebound } \\
\text { agoraphobics with } \\
\text { panic disorder }\end{array}$ & $\begin{array}{l}\text { Telephone } \\
\text { screening }\end{array}$ & 23 & $\begin{array}{l}\text { BDI, FQ, PT, } \\
\text { GP, SA, GI }\end{array}$ & $\begin{array}{l}\text { Baseline, } 2 \mathrm{w}, 4 \\
w, 6 w, 8 w, 10 \\
w, 12 w, 20 w, \\
32 w\end{array}$ & $\begin{array}{l}78 \% \text { at } 6 w \\
61 \% \text { at } 32 w\end{array}$ & 22 \\
\hline Miller (2002) & US & $\begin{array}{l}\text { Women with } \\
\text { history of } \\
\text { recurrent/chronic } \\
\text { depression }\end{array}$ & $\begin{array}{l}\text { Ongoing } \\
\text { longitudinal study }\end{array}$ & 30 & $\begin{array}{l}\text { HRSD, GAS } \\
\text { SAS-SR }\end{array}$ & Baseline, $12 \mathrm{w}$ & $80 \%$ at $12 \mathrm{w}$ & 22 \\
\hline Mohr (2000) & US & $\begin{array}{l}\text { Depressed MS } \\
\text { patients }\end{array}$ & $\begin{array}{l}\text { Telephone } \\
\text { screening }\end{array}$ & 32 & POMS & Baseline, $8 \mathrm{w}$ & $72 \%$ at $8 w$ & 22 \\
\hline Mohr (2005) & US & $\begin{array}{l}\text { Depressed } \\
\text { primary care } \\
\text { patients with MS }\end{array}$ & $\begin{array}{l}\text { MS case registers } \\
\text { \& MS society } \\
\text { newsletters }\end{array}$ & 127 & $\begin{array}{l}\text { BDI, HDRS, } \\
\text { PANAS }\end{array}$ & $\begin{array}{l}\text { Baseline, } 8 \mathrm{wk} \text {, } \\
16 \mathrm{w}, 3 \mathrm{~m}, 6 \mathrm{~m} \text {, } \\
9 \mathrm{~m}, 12 \mathrm{~m}\end{array}$ & $91 \%$ at $16 w$ & 31 \\
\hline Nelson (2003) & US & $\begin{array}{l}\text { Depressed } \\
\text { children aged 8-14 } \\
\text { yrs }\end{array}$ & Not clear & 38 & $\begin{array}{l}\text { K-SADS-P, } \\
\text { CDI }\end{array}$ & Baseline, $8 \mathrm{w}$ & $74 \%$ at $8 w$ & 13 \\
\hline Simon (2004) & US & $\begin{array}{l}\text { Depressed } \\
\text { primary care } \\
\text { patients }\end{array}$ & $\begin{array}{l}\text { Computer } \\
\text { records of } \\
\text { patients starting } \\
\text { new } \\
\text { antidepressant } \\
\text { treatment }\end{array}$ & 600 & SCL, PHQ & $\begin{array}{l}\text { Baseline, } 6 \mathrm{w}, 3 \\
\mathrm{~m}, 6 \mathrm{~m}\end{array}$ & $89 \%$ at $6 \mathrm{~m}$ & 38 \\
\hline $\begin{array}{l}\text { Swinson } \\
\text { (1995) }\end{array}$ & Canada & $\begin{array}{l}\text { Rural primary care } \\
\text { patients suffering } \\
\text { from panic } \\
\text { disorder with } \\
\text { agoraphobia }\end{array}$ & $\begin{array}{l}\text { GP/family } \\
\text { physician referral }\end{array}$ & 46 & $\begin{array}{l}\text { FQ, STAI-T, } \\
\text { BDI, ASI, SCL- } \\
90\end{array}$ & $\begin{array}{l}\text { Baseline, } 10 \mathrm{w}, 3 \\
\mathrm{~m}\end{array}$ & $\begin{array}{l}91 \% \text { at } 10 \mathrm{w}, \\
76 \% \text { at } 3 \mathrm{~m}\end{array}$ & 20 \\
\hline
\end{tabular}

Key: POMS-Profile of Mood States; BDI-Beck Depression Inventory; HAMD/HRSD-Hamilton Rating Scale for Depression; DHP-Duke Health Profile; PSI-Problem Solving Inventory; SCL-90-Symptom Checklist 90; STAI-T-State-Trait Anxiety Inventory (Trait version); ASI-Anxiety Sensitivity Index; PHQ-Patient Health Questionnaire; SCL-Hopkins Symptom Checklist Depression Scale; IES-Impact of Events Scale; GAS-Global Assessment Score; SAS-SR Social Adjustment Scale; K-SADS-P-Schedule for Affective Disorders \& Schizophrenia for School Age Children-Present Episode; CDI-Children's Depression Inventory; PT-Phobic Targets; GP-Global Phobia; SA Social Adjustment; GI-Global Impression; FQ-Fear Questionnaire; YBOCs-Yale Brown Obsessive Compulsive Disorder Scale; PANAS-Positive \& Negative Affect Scale; SF- I2-Mental \& Physical Composite Scales 
Table 3: Results of meta-analyses

\begin{tabular}{|c|c|c|c|c|c|c|c|}
\hline Intervention & Comparison & Disorder & $\begin{array}{l}\text { Follow } \\
\text {-up } \\
\text { period }\end{array}$ & $\begin{array}{c}\text { No. } \\
\text { Comparisons }\end{array}$ & $\begin{array}{c}\text { Total } \\
\text { Participants }\end{array}$ & $\begin{array}{l}\text { Pooled } \\
\text { Effect } \\
\text { Size }\end{array}$ & $95 \% \mathrm{Cl}$ \\
\hline Remote psychotherapy & Control & Depression & $0-6 \mathrm{~m}$ & 7 & 726 & 0.44 & 0.29 to 0.59 \\
\hline Remote psychotherapy & Control & Anxiety-related & $0-6 \mathrm{~m}$ & 3 & 168 & 1.15 & 0.81 to 1.49 \\
\hline Remote psychotherapy & Face-to-face psychotherapy & Depression & $0-6 \mathrm{~m}$ & 1 & 28 & 0.55 & -0.20 to $1.3 \mathrm{I}$ \\
\hline Remote psychotherapy & Face-to-face psychotherapy & Anxiety-related & $0-6 \mathrm{~m}$ & 1 & 63 & -0.11 & -0.60 to 0.38 \\
\hline $\begin{array}{l}\text { Remote psychotherapy } \\
\text { (problem solving therapy) }\end{array}$ & $\begin{array}{l}\text { Remote psychotherapy } \\
\text { (stress management) }\end{array}$ & Depression & $0-6 \mathrm{~m}$ & 1 & 18 & 0.38 & -0.56 to 1.32 \\
\hline $\begin{array}{l}\text { Remote psychotherapy } \\
\text { (cognitive behavioral } \\
\text { therapy) }\end{array}$ & $\begin{array}{l}\text { Remote psychotherapy } \\
\text { (supportive emotion focused } \\
\text { therapy) }\end{array}$ & Depression & $0-6 \mathrm{~m}$ & 1 & 122 & 0.39 & 0.04 to 0.74 \\
\hline $\begin{array}{l}\text { Remote psychotherapy } \\
\text { (cognitive behavioral } \\
\text { therapy) }\end{array}$ & $\begin{array}{l}\text { Remote psychotherapy } \\
\text { (supportive emotion focused } \\
\text { therapy) }\end{array}$ & Depression & $7 \mathrm{m+}$ & 1 & 117 & 0.25 & -0.12 to 0.62 \\
\hline $\begin{array}{l}\text { Remote psychotherapy } \\
\text { (exposure therapy) }\end{array}$ & $\begin{array}{l}\text { Remote psychotherapy } \\
\text { (relaxation therapy) }\end{array}$ & Anxiety-related & $0-6 \mathrm{~m}$ & 1 & 18 & 1.10 & 0.10 to 2.10 \\
\hline $\begin{array}{l}\text { Remote psychotherapy } \\
\text { (exposure therapy) }\end{array}$ & $\begin{array}{l}\text { Remote psychotherapy } \\
\text { (relaxation therapy) }\end{array}$ & Anxiety-related & $7 \mathrm{m+}$ & 1 & 14 & 1.22 & 0.06 to 2.38 \\
\hline
\end{tabular}

Note: Effect size has been coded so that a positive effect size indicates a study where patients in the intervention group have better outcomes than those in the comparison

month follow-up in favor of face-to-face therapy over treatment as usual or waiting list controls [36].
For comparative purposes, meta analyses of lower intensity psychological interventions (e.g. guided self help) for anxiety and depressive disorders report mean effect sizes of between 0.40 and 1.19 [30,31,33,34,36,61], However,

\section{Std diff in means and $95 \% \mathrm{C}$}

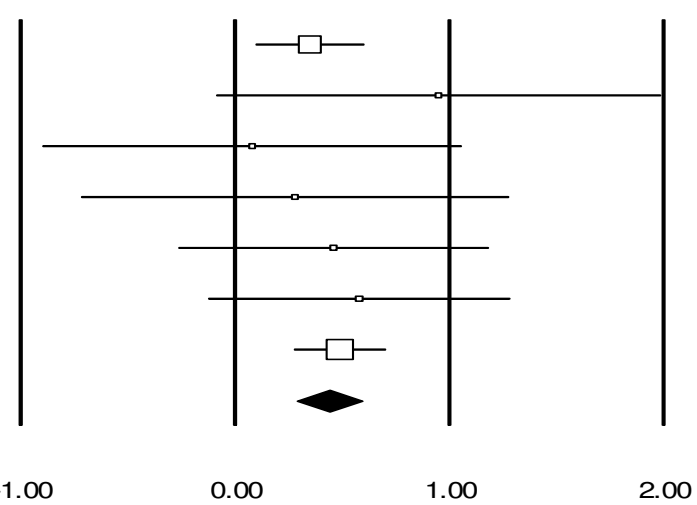

Meta Analysis

Figure I

Analysis of technology-mediated therapy versus control (depression). 


\begin{tabular}{lrrrrr} 
Study name & & \multicolumn{4}{c}{ Statistics for each study } \\
\cline { 3 - 5 } & $\begin{array}{c}\text { Std diff } \\
\text { in means }\end{array}$ & $\begin{array}{c}\text { Lower } \\
\text { limit }\end{array}$ & $\begin{array}{c}\text { Upper } \\
\text { limit }\end{array}$ & p-Value \\
Lange 2001 & 0.80 & -0.02 & 1.62 & 0.06 \\
Lange 2003 & 1.34 & 0.89 & 1.79 & 0.00 \\
Swinson 1995 & 0.98 & 0.33 & 1.63 & 0.00 \\
& 1.15 & 0.81 & 1.49 & 0.00
\end{tabular}

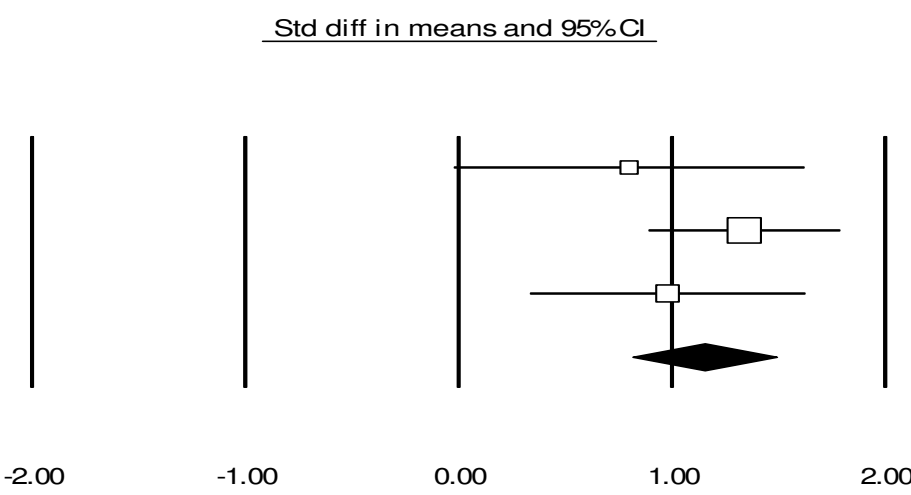

Favours Control

Meta Analysis

\section{Figure 2}

Analysis of technology-mediated therapy versus control (anxiety).

these reviews include studies of treatments that, by definition, use technology to reduce rather than mediate professional therapist input. The effects of such interventions have been shown to be greater for some disorders than others [31,33], with certain problems (e.g. anxiety disorders) responding better to self-help packages supported by increased therapist contact $[30,33]$. The characteristics of patients accessing such treatments are likely to differ substantially from those requiring more traditional and higher intensity therapies, not least in terms of their problem severity and/or motivation for treatment.

A definitive conclusion as to the relative efficacy of technology-mediated versus face-to-face administered psychotherapy can only be drawn from randomized controlled trials comparing the two interventions. A comparison of the efficacy of technology-mediated versus face-to-face psychotherapy was conducted within the present review, with a large but non-significant effect size of 0.55 for depression and a smaller difference of -0.11 for anxietyrelated disorders being obtained. However, a shortage of literature limits the utility of this result. Only two of the 13 studies included in the review directly compared the efficacy of face-to-face psychotherapy with an equivalent intervention delivered via more remote means. It should be acknowledged that even in these studies, meaningful comparisons may be difficult. The ability for remote therapy to overcome multiple physical, psychological and geographical constraints means that the clinical popula- tions who may wish to access such treatments may differ markedly from those in face-to-face services.

Ultimately, the published evidence base for clinical effectiveness of psychotherapies delivered solely or largely via remote communication methods is limited, both in size and quality. Therefore, the effect size estimates reported in the current review can at best only be viewed as preliminary. The majority of identified studies were of relatively small sample size with common shortcomings noted in relation to allocation procedures, identification of primary outcome measures and statistical analyses. From a possible maximum score of 42 , overall quality ratings for the included studies ranged from 13 to 38 with a median of 22 ( $\mathrm{SD}=7.33$ ). Despite the above limitations, these scores remain slightly higher than those observed elsewhere, a recent meta-analysis of brief psychological treatments for depression reporting a mean score of 19 (SD 7.3) for 63 controlled trials assessed against the same quality criteria [36]. Given that the financial implications of remote therapy are likely to be a key factor of interest, it is disappointing that no economic data were available.

Attrition rates for the studies ranged from 9 to $73 \%$ depending upon the population studied, the nature of the intervention and the length of the follow-up period. This heterogeneity suggests that whilst technology-mediated psychotherapies have the potential to be more effective than delayed or usual care, different treatment modalities 
may differ in their perceived acceptability. Data from earlier systematic reviews suggest that attrition rates of alternative models of treatment delivery can also vary widely. Randomized trials of face-to-face therapy for depression report post-treatment drop-outs of between 0 and 60\% [7]. Trials of written self help materials for anxiety and depressive disorders report comparable figures of between 0 and 61\% [62], although attrition from open access online therapy is generally assumed to be higher and may be as much as 99\% [63], Once again however, differences in study context, recruitment and sample characteristics can often prevent any direct comparison of attrition rates across different modes of treatment.

Four of the studies included in the review provided a quantitative measure of patient satisfaction, all indicating a preference for, or equivalent satisfaction with, technologically-mediated care as compared to face-to face delivery $[56,57]$ or usual care $[51,55]$. Two other studies also provided data to suggest that levels of patient satisfaction with remote psychotherapy are high, although in these instances no comparable data from control conditions were available $[48,53]$. Eighty-three per cent of participants in one trial expressed a favorable attitude towards the use of the telephone to deliver psychotherapy and $75 \%$ expressed a desire to continue treatment either now in the future through this medium [53]. However, whether or not these preferences remain consistent across different technologies and communication modalities is unclear. The vast majority of studies included in the present review focused on therapy delivered via the telephone, with very little research effort being directed towards newer and increasingly available resources such as the web. Only one study in the original review examined the efficacy of psychotherapy delivered via videoconferencing and only two studies used the internet, both of which were conducted by the same research team.

A scoping search of literature published since the original searches were undertaken has returned only a small number of additional studies [64-66]. One presents data already included in the present review [64]. Another, examining telephone psychotherapy for depression and conducted as a follow-up to one of trials in the present meta-analysis reports maintenance of clinical benefits when compared to usual care [65]. A third trial that has recently compared face-to-face therapy with a comparable intervention delivered via telepsychiatry reports no significant differences in effect [66].

Whilst the telephone undoubtedly confers specific advantages in terms of its widespread availability and ease of operation, research suggests that videoconferencing may also be particularly suited to psychotherapeutic use. A recent systematic review has shown real time telepsychia- try to represent a highly feasible method of conducting mental health treatments and assessments, often with clinical outcomes and rates of attendance equivalent to those obtained face to face [67]. However, criteria for inclusion and exclusion in this review were not explicitly stated and therefore it is unclear whether or not these observations are based on high quality evidence from randomized trials.

The present review has its limitations. It includes studies examining the effectiveness of psychotherapy delivered via remote communication technology on a scheduled and repeated one-to-one basis and as such, excludes trials examining the effectiveness of more minimal, technologybased interventions. A number of reviews have already been published examining the clinical effectiveness of self-help treatments delivered via technology, and thus the decision was made to conduct a more focused review of optimal relevance to more traditional therapy formats. Nevertheless, publication bias is a problem for any review of controlled trials and given the high dependence on electronic database searching it cannot be certain that unpublished studies do not exist. Due to the small number of studies eligible for the review, funnel plots to assess publication bias were not created in line with recommendations [68]. The analysis treated 'usual care' and waiting list' controls as equivalent to 'no treatment', although it should be noted that previous studies have suggested that effects in usual care may be significantly lower [69]. Translating dichotomous outcomes into standardised effect sizes for the purposes of the meta analysis does allow the most comprehensive summary of the outcome data, but these transformations are based on assumptions which may not always be warranted.

\section{Conclusion}

Technology-mediated psychotherapy provision has the potential to overcome many of the barriers to care associated with more traditional face-to-face interventions. Data suggest that good treatment effects and by implication therapeutic alliance may not be dependent on a patient and therapist being co-located. However, the limited amount of evidence that is available and the restricted number of studies in any one subgroup as yet prevents any definitive conclusions from being drawn. Future research priorities should include overcoming the methodological shortcomings of published work by conducting large-scale trials that incorporate both clinical outcome and more process-orientated measures. In particular, these studies should seek to compare remote psychotherapy with more conventional face-to-face methods by quantifying levels of patient and therapist satisfaction and exploring the potential impact of different modes of communication on therapeutic outcome and examining the quality of the therapeutic alliance that is established, 


\section{Competing interests}

The authors declare that they have no competing interests.

\section{Authors' contributions}

PEB participated in the study design, search strategy development, retrieval of articles, screening of articles, quality assessment, data extraction, data synthesis, and writing of manuscript. PB participated in the study design, quality assessment, data extraction, meta-analysis, data synthesis, and writing of manuscript. KL, SG, DR and LG participated in the study design, quality assessment, data extraction, and writing of manuscript. PBarnes participated in the retrieval of articles, screening of articles, quality assessment, data extraction, and writing of manuscript. All authors read and approved the final manuscript.

\section{Appendix}

Electronic search strategy

1. online therapy/or telemedicine/or Diagnosis, Computer-Assisted/or Therapy, Computer-Assisted/

2. telecommunications/or telephone/or videoconferencing/or teleconferencing/

3. internet/or computer assisted instruction/or virtual reality/or Electronic Mail/

4. phone\$.mp. or telephone\$.mp. or telecommnicat\$.mp

5. (text and messag\$).mp. or SMS.mp. or (short and messag\$).mp.

6. videophone\$.mp. or videoconferenc\$.mp. or teleconferenc\$.mp

7. email.mp. or (electronic and mail).mp. or (electronic and communication).mp.

8.(virtual and reality).mp. or VR.mp.

9.(bulletin and board\$).mp. or (discussion and board\$).mp. or (discussion and list\$).mp.

10. internet.mp. or online.mp. or worldwide web.mp. or web based.mp.

11. Emedicine.mp. or Etherapy.mp. or Ehealth.mp.

12. telemedicin\$.mp. or teleconsult\$.mp. or telepsychiatry.mp. or telehealth.mp. or teletherap\$.mp.

13. (computer and based).mp.or (computer and mediated).mp. or (computer and assisted).mp.
14. (remote and consultation).mp.

15. 1 or 2 or 3 or 4 or 5 or 6 or 7 or 8 or 9 or 10 or 11 or 12 or 13 or 14

16. Psychotherapy/or Brief psychotherapy or Individual Psychotherapy/or Psychotherapeutic Techniques/or Supportive Psychotherapy or or Group Psychotherapy/or Psychotherapeutic Processes/

17. psychotherap\$.mp. or psychodynamic therap\$.mp. or PDT.mp. or psychoanalytic therap\$.mp.

18. Behavior Therapy/or Cognitive Therapy/or Cognitive Behavior Therapy/or Rational Emotive Behavior Therapy/ or Behavior Modification/

19. cognitive behavio? $\mathrm{r}$ therap\$.mp. or cognitive therap\$.mp. or behavio?r therap\$.mp. or CBT.mp. or cognitive analytic therap\$.mp. or behavio? $\mathrm{r}$ modification.mp.

20. Relaxation Therapy/or relaxation therap\$.mp.

21. Family Therapy/or Interpersonal Psychotherapy/or Psychodynamic Psychotherapy/or Marital Therapy or Group therapy/

22. Interpersonal therap\$.mp or IPT.mp.

23. family therap $\$ . m p$. or marital therap $\$ . m p$. or group therap\$.mp. or support group\$.mp.

24. GROUP COUNSELING/Or PSYCHOTHERAPEUTIC COUNSELING/or EDUCATIONAL COUNSELING/or COUNSELING/or MARRIAGE COUNSELING/or COUNSELING PSYCHOLOGY/or PARENT COUNSELING/or FAMILY COUNSELING/or PATIENT COUNSELING/

25. counsel?ing.mp.

26. Self Help/or Self Management/or exp Self Help Techniques/

27. self treatment.mp. or self help.mp. or self directed.mp. or self management.mp.

28. Behavior Modification/or Relaxation Techniques/or Relaxation Training/

29. relaxation training.mp. or relaxation techniques.mp.

30. psychosocial support.mp. or psychological therap\$.mp. or psychological treatment\$.mp. 


\section{1. exposure therapy.mp.}

32. 16 or 17 or 18 or 19 or 20 or 21 or 22 or 23 or 24 or 25 or 26 or 27 or 28 or 29 or 30 or 31

\section{15 and 32}

\section{4. limit 33 to English}

\section{5. remove duplicates from 34}

\section{References}

I. Murray CJL, Lopez AD, (Eds): The global burden of disease and injury series, A comprehensive assessment of mortality and disability from diseases, injuries and risk factors in 1990 and projected to 2002. Volume I. Cambridge, MA: Harvard School of Public Health on behalf of the World Health Organization and the World Bank: Harvard University Press; 1996.

2. American Psychiatric Association (APA): Practice guidelines for major depressive disorder in adults. Am J Psychiatry 1993, I 50:SI-S26.

3. Department of Veteran Affairs (DVA): Clinical guideline for major depressive disorder. Washington, DC: Veterans Health Administration; 1993.

4. Depression Guideline Panel: Depression in primary care: treatment of major depression. Rockville, Md.: US Dept. of Health and Human Services, Public Health Service, Agency for Health Care Policy and Research; 1993.

5. American Psychiatric Association (APA): Practice guideline for the treatment of patients with panic disorder. Am J Psychiatry 1998, I 55: I-34.

6. Young A, Klapp R, Sherbourne C, Wells K: The quality of care for depressive and anxiety disorders in the United States. Arch Gen Psychiatry 200I, 58:55-6I.

7. Brody DS, Khaliq AA, Thompson TL: Patients' perspectives on the management of emotional distress in primary care settings. J Gen Intern Med 1997, I 2:403-406.

8. Alvidrez J, Azocar F: Distressed women's clinic patients: preferences for mental health treatments and perceived obstacles. Gen Hosp Psychiatry 1999, 2 I:340-347.

9. Sirey J, Bruce M, Alexopoulos G, Perlick D, Friedman S, Meyers B Stigma as a barrier to recovery: perceived stigma and patient-rated severity of illness as predictors of antidepressant drug adherence. Psychiatric services 52:1615-1620.

10. Hollon S, Munoz RF, Barlow DH, Beardslee WR, Bell CC, Bernal G, Clarke GN, Franciosi LP, Kazdin AE, Kohn L, Linehan MM, Markowitz IC. Persons JB, Niederehe G, Sommers D: Psychosocial intervention development for the prevention and treatment of depression: promoting innovation and increasing access. Biological psychiatry 2002, 52:610-630.

II. Nutting PA, Rost K, Dickinson M, Werner JJ, Dickinson P, Smith JL, Gallovic B: Barriers to initiating depression treatment in primary care practice. I Gen Intern Med 2002, I 7: I03- I I I.

12. Nickleson D: Telehealth and the evolving health care system: strategic opportunities for professional psychology. Prof Psychol Res Pr 1998, 29:527-535.

13. Maheu M, Whitten P, Allen A: E-health, telehealth and telemedicine. San Francisco, CA: Jossey-Bass; 2001.

14. FCC: Supplemental telephone penetration report. US Federal Communications Commission; 1993.

15. VandenBos GR, Williams S: The internet versus the telephone: what is telehealth anyway? Prof Psychol Res Pr 2000, 3 I:490-492.

16. Veterans Health Administration (VHA): Telemental health toolkit. Veterans Health Administration; 2003.

17. Smith MA, Senior C: The internet and clinical psychology: a general review of the implications. Clin Psychol Rev 2001, 2I:129-I36.

18. Horvath AO, Bedi RP: The alliance. In In Psychotherapy relationships that work: therapist contributions and responsiveness to patients Edited by: Norcross JC. New York: Oxford University Press; 2002.

19. Wootton R, Yellowlees P, McLaren P: Telepsychiatry and E-Mental Health. London: Royal Society of Medical Press; 2003.
20. Kraut R, Patterson M, Lundmark V, Kiesler S, Mukopadhyay T, Scherlis $W$ : Internet paradox: a social technology that reduces social involvement and psychological wellbeing? Am Psychol 1998, 53:1017-1031.

21. Stamm BH: Clinical applications of telehealth in mental health care. Prof Psychol Res Pr 1998, 29:536-542.

22. Hsuing R: E-therapy. London: WW Norton \& Company Ltd; 2002.

23. Simon G, Von Korff M, Rutter C, Wagner E: Randomised trial of monitoring, feedback, and management of care by telephone to improve treatment of depression in primary care. BMJ 2000, 320:550-554.

24. Datto C, Thompson R, Horowitz D, Disbot M, Oslin D: The pilot study of a telephone disease management for depression. Gen Hosp Psychiatry 2003, 25: I69-I77.

25. Oslin D, Sayers S, Ross J, Kane V, Ten Have T, Conigliaro J, Cornelius $\mathrm{J}$ : Disease management for depression and at-risk drinking via telephone in an older population of veterans. Psychosomatic medicine 2003, 65:93|-937.

26. Fortney JC, Pyne JM, Edlund MJ, Robinson DE, Mittal D, Henderson $\mathrm{KL}$ : Design and implementation of the telemedicineenhanced antidpressant management study. Gen Hosp Psychiatry 2006, 28:18-26.

27. Richards DA, Lankshear AJ, Fletcher J, Rogers A, Barkham M, Bower P, Gask L, Gilbody S, Lovell K: Developing a U.K. protocol for collaborative care: A qualitative study. Gen Hosp Psychiatry 2006, 28:296-305

28. Kaltenthaler E, Parry G, Beverley C: Computerized cognitive behaviour therapy: A systematic review. Behavioural \& cognitive psychotherapy 2004, 32:31-55.

29. Kaltenthaler E, Brazier J, De Nigris E, Tumur I, Ferriter M, Beverley C, Parry G, Rooney G, Sutcliffe P: Computerized cognitive behaviour therapy for depression and anxiety update: A systematic review and economic evaluation. Health Technology Assessment 2006, 10:1-70.

30. Scogin F, Bynum J, Stephens G, Calhoon S: Efficacy of self-adminstered treatment programs: meta-analytic review. Prof Psychol Res $\operatorname{Pr} 1990,21: 42-47$.

31. Gould R, Clum G: A meta-analysis of self help treatment approaches. Clinical psychology review 1993, I3:169-186.

32. Christensen A, Jacobson N: Who (or what) can do psychotherapy? The status and challenge of nonprofessional therapies. Psychological science 1994, 5:8-14.

33. Marrs R: A meta-analysis of bibliotherapy studies. Am J Community Psychol 1995, 23:843-870.

34. Spek V, Cuijpers P, Nyklicek I, Riper H, Keyzer J, Pop V: Internetbased cognitive behaviour therapy for symptoms of depression and anxiety: a meta-analysis. Psychological medicine 2007, 37:319-328.

35. Higgins JP, Green S: Cochrane handbook for systematic reviews of interventions 4.2.5. In The Cochrane Library Issue 3 Chichester UK: John Wiley \& Sons Ltd; 2005.

36. Churchill R, Hunot V, Corney R, Knapp M, McGuire H, Tylee A, Wessely S: A systematic review of controlled trials of the effectiveness and cost-effectiveness of brief psychological treatments for depression. Health Technol Assess 2001, 5(35): $1-173$.

37. World Health Organisation: The ICD-I 0 classification of mental and behavioural disorders: clinical descriptions and diagnostic guidelines. Geneva: World Health Organisation; 1992

38. American Psychiatric Association (APA): Diagnostic and statistical manual of mental disorders (DSM-IV). Washington, DC: American Psychiatric Association; 1994.

39. Moncrieff J, Churchill R, Drummon C, McGuire H: Development of a quality assessment instrument for trials of treatments for depression and neurosis. Int J Methods Psychiatr Res 200I, 10:126-133

40. Jadad A: Randomised Controlled Trials: A User's Guide. London: BMJ Books; 1998.

4I. Metaanalysis [http://www.metaanalysis.com]

42. Cohen J: Statistical power analysis for the behavioural sciences. New Jersey: Lawrence Erlbaum; 1988.

43. Sutton A, Abrams K, Jones D, Sheldon T, Song F: Systematic reviews of trials and other studies. Health Technology Assessment 1998, 2: 1-276

44. Higgins JP, Thompson SG, Deeks J], Altman DG: Measuring inconsistency in meta-analyses. BMJ 2003, 327:557-560. 
45. Deeks J], Higgins JPT, Altman DG: Analysing and presenting results. In Cochrane Handbook for Systematic Reviews of Interventions 4.2.5 Edited by: Higgins JPT, Green S. Chichester UK: John Wiley \& Sons Ltd; 2005.

46. Lipsey M, Wilson D: Practical Meta-Analysis. Newbury Park: Sage; 2001.

47. Swinson R, Fergus K, Cox B, Wickwire K: Efficacy of telephoneadministered behavioral therapy for panic disorder with agoraphobia. Behav Res Ther 1995, 33:465-469.

48. Lange A, Ven JP van de, Schrieken B, Emmelkamp PMG: Interapy: Treatment of posttraumatic stress through the Internet: a controlled trial. J Behav Ther Exp Psychiatry 200I, 32:73-90.

49. Lange A, Rietdijk D, Hudcovicova M, Ven JP van de, Schrieken B, Emmelkamp PMG: Interapy: A controlled randomized trial of the standardized treatment of posttraumatic stress through the Internet. J Consult Clin Psychol 2003, 71:90I-909.

50. Lynch D, Tamburrino M, Nagel R: Telephone counseling for patients with minor depression: preliminary findings in family practice setting. Journal of family practice 1997, 44:293-296.

5I. Hunkeler E, Meresman J, Hargreaves W, Fireman B, Berman W, Kirsch A, Groebe J, Hurt S, Braden P, Getzell M, Feigenbaum P, Peng T, Salzer M: Efficacy of nurse telehealth care and peer support in augmenting treatment of depression in primary care. Arch Fam Med 2000, 9:700-708.

52. Mohr D, Likosky W, Bertagnolli A, Goodkin D, Wende J Van Der, Dwyer P, Dick L: Telephone-administered cognitive-behavioural therapy for the treatment of depressive symptoms in multiple sclerosis. J Consult Clin Psychol 2000, 68:356-36I.

53. Miller L, Weissman M: Interpersonal psychotherapy delivered over the telephone to recurrent depressives: a pilot study. Depress Anxiety 2002, 16: I 14- I I7.

54. Lynch D, Tamburrino M, Nagel R, Smith N: Telephone-based treatment for family practice patients with mild depression. Psychol Rep 2004, 94:785-792.

55. Simon G, Ludman E, Tutty S, Operskalski B, Von Korff M: Telephone psychotherapy and telephone care management for primary care patients starting antidepressant treatment: A randomized controlled trial. JAMA 2004, 292:935-942.

56. Lovell K, Cox D, Haddock G, Jones C, Raines D, Garvey R, Roberts $C$, Hadley S: Telephone administered cognitive behaviour therapy for treatment of obsessive compulsive disorder: randomised controlled non-inferiority trial. BMJ 2006 333:883-887.

57. Nelson E, Barnard M, Cain S: Treating childhood depression over videoconferencing. Telemed J E Health 2003, 9:49-55.

58. McNamee G, O'Sullivan G, Lelliot P, Marks IA: Telephone-guided treatment for housebound agoraphobics with panic disorder: Exposure vs. Relaxation. Behavior therapy 1989, 20:49|-497.

59. Mohr D, Hart SL, Julian L, Catledge C, Honos-Webb L, Vella L, Tasch E: Telephone-administered psychotherapy for depression. Arch Gen Psychiatry 2005, 62:1007-1014.

60. Wampold BE, Mondin GW, Moody M, Stich F, Benson K, Ahn H: A meta-analysis of outcome studies comparing bona fide psychotherapies: empirically 'all must have prizes'. Psychological bulletin 1997, I 22:203-2I5.

6I. Den Boer P, Wiersma D, Bosch R Van Den: Why is self-help neglected in the treatment of emotional disorders? A metaanalysis. Psychological medicine 2004, 34:959-97I.

62. Bower P, Rlchards D, Lovell K: The clinical and cost-effectiveness of self-help treatments for anxiety and depressive disorders in primary care: a systematic review. Br J Gen Pract 200I, 5 I:838-845.

63. Eysenbach G: The law of attrition. J Med Internet Res 2005, 7:el I.

64. Nelson E, Barnard M, Cain S: Feasibility of telemedicine intervention for childhood depression. Counselling and psychotherapy research 2006, 6(3): 191-195.

65. Ludman EJ, Simon GE, Tutty S, Von Korff : A randomized trial of telephone psychotherapy and pharmacotherapy for depression: continuation and durability of effects. J Consult Clin Psychol 2007, 75(2):257-266.

66. De Las Cuevas C, Arredondo MT, Cabrera MF, Sulzenbacher H, Meise U: Randomized clinical trial of telepsychiatry through videoconference versus face to face conventional psychiatric treatment. Telemed J E Health 2006, I 2(3):34|-350.

67. Norman S: The use of telemedicine in psychiatry. I Psychiat Ment Health Nurs 2006, 1 3:71 I-777.
68. Egger M, Davey-Smith G, Schneider M, Minder C: Bias in metaanalysis detected by a simple graphical test. BMJ 1997, 31 5:629-634.

69. Gellatly J, Bower P, Hennessy S, Richards D, Gilbody S, Lovell K: What makes self-help interventions effective in the management of depressive symptoms? Meta-analysis and metaregression. Psychological medicine 2007, 37(9): | 2|7-| 228.

\section{Pre-publication history}

The pre-publication history for this paper can be accessed here:

\section{http://www.biomedcentral.com/1471-244X/8/60/pre} pub
Publish with Bio Med Central and every scientist can read your work free of charge

"BioMed Central will be the most significant development for disseminating the results of biomedical research in our lifetime. "

Sir Paul Nurse, Cancer Research UK

Your research papers will be:

- available free of charge to the entire biomedical community

- peer reviewed and published immediately upon acceptance

- cited in PubMed and archived on PubMed Central

- yours - you keep the copyright
BioMedcentral 